\section{Absence of Electrical Activity of the Pigeon's Pineal Organ in Response to Light}

Recently, environmental illumination has been shown to affect the enzymatic activity required for the synthesis of melatonin in the pineal organ of rat and hen ${ }^{1,2}$. Moreover, illumination of young ducks resulted in acceleration of the development of sexual organs. Since this has been found both before and after removal of the lateral eyes, it has been concluded that part of the brain itself may be sensitive to light ${ }^{3}$. In order to obtain direct evidence of light sensitivity of the avian pineal organ comparable to that of lower vertebrates ${ }^{4-6}$, the epiphysis cevebri of 8 adult pigeons, Columba livia, was surgically exposed under urethane anaesthesia and systematically explored by microelectrodes ${ }^{7}$ inserted into the organ. With all animals investigated the evidence was entirely negative, i.e. direct illumination of the pineal organ or of the lateral eyes did not produce any electrical activity related to the onset or the cessation of light. This result cannot be due to technical reasons since, when the tip of the electrode was pushed deeper into the pineal organ, a sustained train of impulses of small size was seen which did not respond to illumination ${ }^{8}$. With a somewhat more backward and deeper position of the electrode, large spike potentials of the cerebellum were observed. The evident lack of electrical activity of the pigeon's pineal organ in response to light corresponds to recent electron microscopic findings, according to which synapses and typical photoreceptor cells with regular outer segments in the pigeon and in the duck are absent ${ }^{9}$.

Zusammenfassung. Im Gegensatz zur Epiphyse von Fischen, Amphibien und Reptilien erweist sich das Pinealorgan der Taube bei Untersuchung mit Einstichelektroden als nicht lichtempfindlich.

Y. MORITA

William G. Kerckhotf-Herzforschungsinstitut der Max-Planck-Gesellschaft, Bad Nauheim (Germany), March 28, 1966.

1 R. J. Wurtman, J. Axelrod, and L. S. Phillips, Science 142, 1071 (1963).

2 J. Axelrod, R. J. Wurtman, and Ch. M. Winget, Nature 201, 1134 (1964).

3 J. Benoit and I. Assenmacher, Recent Prog. Horm. Res. 15, $143(1959)$.

4 E. Dodt and E. Heerd, J. Neurophysiol. 25, 405 (1962).

5 W. H. Miller and M. L. Wolbarsht, Science 135, 316 (1962).

6 E. Dodt, Experientia 19, 642 (1963).

7 Y. Morita, Pflügers Arch. ges. Physiol. 286, 97 (1965).

8 The position of the tip of the recording electrode was identified to be close to a bundle of unmedullated fibres in the basal part of the pineal organ. A full account of this work will appear elsewhere.

9 A. OKSChe und M. VAupel-von Harnack, Z. Zellforsch. 69, 41 (1966).

\section{Differentiation of the Epithelium in Early Grafts of the Mouse Müllerian Vaginal Region}

Recent investigations ${ }^{2-4,8}$ have disclosed a dual origin of the mouse vaginal epithelial lining: the anterior part from the muillerian epithelium, the posterior part from the sinus epithelium.

In an earlier paper, FonsBERG ${ }^{3}$ reported studies on the differentiation of the epithelium in homografts of the vaginal müllerian epithelium from new-born female mice that were allowed to grow intramuscularly in female litter-mates. The results were interpreted as excluding any contribution of the sinus epithelium at the differentiation of the epithelium in the anterior part of the vagina, a thing that was formerly widely believed to occur. However, apart from a cellular contribution, an inductive stimulus from the sinus epithelium on the müllerian epithelium could not be excluded. Another possibility is that the mesenchyme plays some role in this connection (GRobsteIN ${ }^{5}$ ). In this investigation, we wish, among other things, to throw light on this problem by using early fetal grafts of the mouse muillerian vaginal epithelium.

Material and methods. Albino mice from a closed stock were used in this investigation. At a gestational age of 14.5 days, calculated from the time of an observed spermal plug, the pregnant mother animal was killed and the fetuses removed. Under a dissecting microscope, the anterior part of the müllerian vaginal region was removed for transplantation (see Figure 1). The rest of the fetuses were fixed in Bouin's fluid, paraffin embedded, and sectioned transversely in $5 \mu$ sections. These were stained in hematoxylin and eosin. The donor fetuses were studied in order to exclude any contamination of sinus epithelium in the grafts. In some cases, a piece of one of the fetal uterine horns was also removed for transplantation.

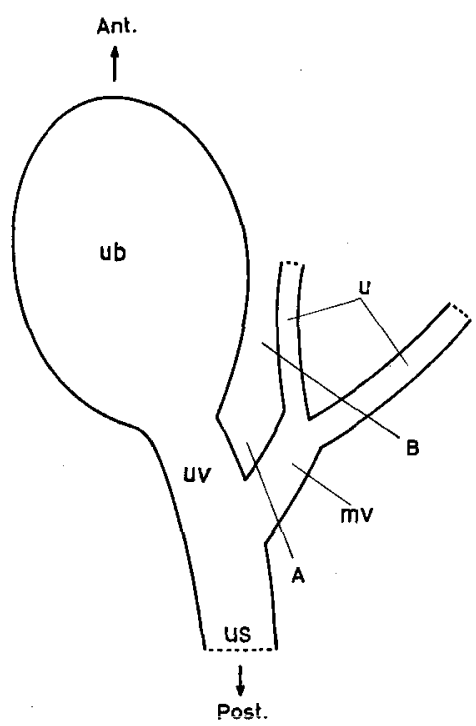

Fig. 1. Schematic presentation of the müllerian vaginal region as seen in the dissecting microscope in a 14.5 day fetus. mv, müllerian vagina; $u$, uterine horn; ub, urinary bladder; ur, urethra; us, urogenital sinus. The mülerian vagina was cut at $A$ and the uterine horns at $B$. The region between $A$ and $B$ was transplanted into newborn female mice. 